\title{
Building Holistic Evidence for Social Media Impact
}

\begin{abstract}
Social media measurement is important for understanding an organization's reach and engagement with its audiences. In response to Warren Kagarise and Staci M. Zavattaro's question about what works in social media measurement, this article discusses how public administration researchers and practitioners are using social media data that they can easily collect from social media platforms and contrasts these practices with data measurement efforts that can provide deeper insights for evidence-based decision making. This evidence includes interactivity and connectivity among citizens, attributes of network actors, and network structures and positions to understand how content travels through the network and who are the influential actors.
\end{abstract}

$\mathrm{O}$ rganizations are using free analytics provided by social media platform providers, such as the number of followers and the number of times a post was seen, as an indicator of the quality of and interest in a topic. Others use the number of times an update was shared as a proxy for reach and engagement, as presented in this issue of Public Administration Review by Warren Kagarise and Staci Zavattaro in their case study of the City of Issaquah, Washington ("Social Media: How One City Opens the Evidence Black Box"). Scholars use the same publicly available measures to make inferences about degrees of participation, transparency, or even democratization and legitimacy of government (see, e.g., Grimmelikhuijsen and Meijer 2015). What current practices neglect are measures that can be derived from the characteristics of the social networking platforms and the types of interactions that citizens have among themselves and with the social media content. These measures use dyadic relationships between actors, their degree of interactivity, and the resulting structures and positions, which can be analyzed to draw conclusions about the segmentation of stakeholders or the quality of content shared on social media sites. Lastly, what is missing in current practices and conversations about social media measurement and its impact is the link to organizational mission and potential innovative outcomes of the knowledge gained using analysis and interpretation. As Isett, Head, and VanLandingham (2016) suggest, additional data collection and analytical approaches are needed to provide evidence for decision making.

In response to Kagarise and Zavattaro's measurement approach, this article discusses how current social media measurement techniques are represented in the public administration and government technology literature, describes how scholars use publicly available social media data sources, and derives a series of open research questions for public administration scholars and practitioners that are not answered by the current use of social media data.

\section{The State of Social Media Measurement in the Public Sector}

Kagarise and Zavattaro provide two measures of social media impact: breadth and depth of citizen engagement with the city's social media content. First, they measure breadth as an indicator of the increase in organizational awareness among constituents.

They use indicators such as the number of followers, number of page views, and number of organizational mentions. Second, the city uses three measures of depth. It measures the depth of follower engagement by counting how often citizens visit, comment, reply to, or add content to the city's social media accounts. Issaquah then measures depth, called "word-ofmouse" engagement, by tracking the number of times city content is shared or tagged or dialogue evolves as a result of a social media post. These measures overlap with the breadth indicators, and it is unclear how the city distinguishes between page views and visits, which are used in both measures. Finally, the city measures depth as an indicator of sentiment with a deep dive into the content of the posts. A form of sentiment analysis is used to indicate whether citizens adopt a positive tone by counting the use of exclamation points, emoji, and photos shared.

The data that Issaquah uses are freely available on social media sites, which are third-party platforms that
Ines Mergel is full professor of public administration in the Department of Politics and Public Administration at the University of Konstanz in Germany. Her research focuses on digital transformation in the public sector.

E-mail: ines.mergel@uni-konstanz.de 
allow users to create a digital profile; share information in the form of text, videos, pictures, and links to external content; subscribe to other users' content; and connect citizens with each other (see, e.g., Boyd and Ellison 2007). The result of online interaction is an interconnected network of social ties between content and actors. Citizens connect with government's social media content or forward it to their own network of friends. This form of interactivity among users with social media content differentiates the one-on-one relationships that citizens usually create with government during a service delivery transaction. Understanding whether an organization has reached a citizen with its social media content is therefore more difficult to observe than a direct face-to-face informational or educational session, where behavioral changes can be observed directly. Traditional metrics and measurement systems quantify an organization's performance. Metrics focus on past interactions by counting the degree of completion, such as the number of closed cases, which is then used to estimate future needs. These so-called predictive analytics help government organizations define goals and strategic direction (Kim, Trimi, and Chung 2014). In addition to these administratively designed data collections and analytics, government is taking social media and other Internet-generated data into account to predict the impact of policies on citizens and to understand behavioral preferences and changes in mood toward government (Daniell, Morton, and Insua 2016; De Marchi, Lucertini, and Tsoukiàs 2016; Mergel, Rethemeyer, and Isett 2016).

The current status of social media measurement reflects the risk-averse context in which governments are interacting and the restrictive nature of collecting and using personalized information that can be gleaned from social media interactions with private citizens. Social media information is therefore mostly pushed out to citizens without engaging citizens in conversations, asking for feedback from them, or allowing agencies to respond to online requests for additional information (Campbell, Lambright, and Wells 2014; Mergel 2013a; Mossberger, Wu, and Crawford 2013). That means that organizations tend to follow an information and education approach toward content sharing and recycle information that has already been published on the website, such as official memos or press releases also on social media (see, e.g., Mergel 2013b for a discussion of social media tactics). Rarely do they ask citizens for individual input, engage them in backand-forth discussions to gain a deeper understanding of citizens' needs, or provide direct feedback to citizen questions or individual requests.

Given these limitations in the use of social media, it is not surprising that public administration research is restricted to measuring direct interactions between government and citizens only and uses proxy measures to evaluate the impact of social media use. In the Issaquah case, the measures are limited to the number of directly countable shares of a post and the number of visits to a site, but they lack an in-depth sentiment analysis of the content of information provided by citizens or a deep dive into discussion threads or they ways in which information provided by government is changing citizen behavior.

Similarly, public administration research either counts the number and types of interactive social media tools listed on a government website or the number of followers, posts, comments, or reshares.
The goals include (1) fulfilling the information and education mandate for which social media tools are seen as a natural extension of government's communication toolkit (Mergel 2013a, 2013b, 2014) and (2) increasing openness and accountability by sharing information through social media (Stamati, Papadopoulos, and Anagnostopoulos 2015). In addition, most social media research is limited to the public micro-blogging service Twitter. Even though Facebook is the most popular social media site (according to a Pew Research Center study, 79 percent of online adults use Facebook; see Greenwood, Perrin, and Duggan 2016), its walled-off nature does not allow researchers to use its application programming interface (API). While Twitter also biases online data collection efforts with arbitrary rate-limit reductions of the allowable data to be downloaded for analysis (Driscoll and Walker 2014), most social media research is conducted using Twitter's API to download, for example, discussion threads in the form of a hashtag. This introduces a bias by limiting insights into the organization's depth and breadth of social media impact to those Internet users who prefer to use Twitter, which captures only 24 percent of the online population.

Some studies dive deeper into the tasks and purposes for which government organizations are using social media tools, but they rarely link to outcomes or measure interactivity beyond the frequencies of updates and interactions.

As an example, Maultasch and Welch (2013) analyze how and why social media tools are used by counting the number of social media tools linked from a government's website, reporting the tasks and purposes.

Similarly, Mossberger, Wu, and Crawford (2013) use a content analysis of government websites, counting the number of interactive tools and showing that governments rarely go beyond pushing out content through their social media channels. Comparative country studies show similar approaches: social media interactions are counted by using either publicly available numbers of followers or posts, but scholars rarely conduct an analysis of one-to-one or oneto-many interactions between government and citizens.

Grimmelikhuijsen and Meijer (2015) use interview and Internet survey data to link the use of Twitter among police departments to positive perceptions of transparency and legitimacy. They do not analyze Twitter use or measure connectivity and interactivity to show how use is linked to the outcome they study; they instead link citizens' searches for police information on social media to citizens' perceptions.

Campbell, Lambright, and Wells (2014) show whether organizations use Facebook, Twitter, or YouTube accounts and how the existence of accounts is linked to increases in awareness among citizens. Using content analysis, they measure the mean number of posts, events per month, likes, and reshares of content as an indicator of reach.

Zavattaro, French, and Mohanty (2015) use a machine-learning approach to conduct a sentiment analysis of the content of tweets and find that a positive tone is related to increased citizen participation with government. 
Porumbescu (2016) focuses on the flip side: the article aims to understand whether citizens are able to find information about government on social media and how this content is aligned with their satisfaction and perception of government's trustworthiness. Warren, Sulaiman, and Jaafar (2014) find a similar link: active use of Facebook is linked to increased trust in institutions and a higher likelihood that citizens are organizing or participating in civic events.

It is especially important for gov-
ernment to pay attention beyond
the so-called echo chamber of
followers who share the organiza-
tion's beliefs and values.

al. 2016). This relational approach aims to conceptualize online interactions in the form of networks that are connected with each other based on common interests or the type of content shared or accessed. It is especially important for government to pay attention beyond the so-called echo chamber of followers who share the organization's beliefs and values. Connections are dynamically developing and serve as conduits through which resources such as (mis)information and influence are spreading. The recent use of big data derived from social media data includes the risk of

These findings are in line with more in-depth analytical approaches to the interactions between organizational postings and reactions from citizens to the content. For example, Wukich and Mergel (2016) find that government organizations rarely reuse social media information beyond their trusted and vetted sources. They measure reuse as the type of information that is retweeted from other government organizations or nonprofits that work in the same space.

Few sources show how social media data are used for decision making. One exception is Bekkers, Edwards, and de Kool (2013), who look into social media monitoring activities by four Dutch government bodies. They use desk research and semistructured interviews to understand the social media monitoring processes, but they do not explain how the agencies are measuring social media use.

A review of the literature shows that diverse attempts to assess social media impact are in place. Managers are generally using the data that are the least burdensome and relatively easy to collect to derive the impact of their online interactions. Others, such as the City of Issaquah, go further by rethinking the use of these data and interpreting social media impact as a combination of different data points to derive the breadth and depth of their online content. The majority of data use is limited to freely available and easily accessible data points that can be downloaded or derived as counts from social media posts. Current efforts at deeper analysis are limited to free, easy, or budget-friendly approaches, but they only scrape the surface in terms of analysis and insights. Deeper analytical approaches are limited to expensive for-profit business analytics software tools that evaluate network interactions or visualize resulting social structures. The next sections identify foundational questions for deeper social media impact analysis and innovative ways to measure the impacts that transcend reliance on data that are accessible only on current platforms.

\section{Open Research Questions for Social Media Analytics}

For public administration scholars and practitioners, it is important to understand, as Kagarise and Zavattaro write, "what works" when measuring the impact of public sector social media activities beyond the number of followers or the number of times content was read or shared. Understanding how information is spread through the network of online connections among an organization's constituency can help the organization understand interests, worldviews, impact, or the constellation of diverse subgroups among the population of followers (Del Vicario et "simple" use of easily accessible social media data, which might overamplify or misrepresent information peaks (Lavertu 2016).

The following questions can serve as a guide for future research by practitioners and scholars:

- How can government organizations continuously understand the network structure of their followers online and shape the network structure of their followers?

- What does the sociodemographic markup of an organization's online constituency look like in comparison with its actual stakeholders? Can the organization reach the subgroups of its constituency, or are some of those who might be important to gain representative insights unreachable online?

- Do government's social media interactions lead to intended behavioral changes? Approaches such as experiments, network, or content analyses help to understand the overlap of offline and online interactions and the impact of content on segments of the network.

- How do the breadth and depth of online interactions lead to improved outcomes for the relationships established online, specifically for administrative decision making, increased service quality, or more efficient and effective government?

- How are insights from social media used to adjust social media tactics and provide performance indicators to change decision making?

- How can existing administratively collected data sets that are designed by government be combined with unstructured social media data to gain insights? How does government have to adjust privacy and security policies?

- Social media data allow government to search for the best these measures be combined with traditional key performance indicators?

\section{Using a Holistic Approach to Measure Social Media Use for Decision Making}

Using social network analytical approaches, relational metrics can be derived from the characteristics of and interactions among citizens and government organizations. Characteristics of online social media sites include two main aspects: users create profile pages in which they include personal identifiers about themselves (such as personal demographic information, including their names and geographic location, and other types of personal information, empirical evidence and to combine data sets. How else can 
including political viewpoints and relationships status) (Boyd and Ellison 2007; Ellison and Boyd 2013; Kane et al. 2014). This voluntary information is labeled "attribute data" and can be used for deeper analyses of the types of citizens who are engaging with content. These data are, for example, summarized for free in Facebook's Page Insights analytics. However, in the public sector there might be limitations on analyzing these data beyond the aggregate level because of privacy concerns or policy limitations of personally identifiable information (NIST 2010).

The features of social media platforms differ from other types of communication tools and result in a dynamic, observable network of followers and their friendship networks - the social graph (Ugander et al. 2011). This "articulated" network of confirmed contacts or follower relationships is accessible to researchers, who can use the number of followers, number of new followers, or number of times followers have shared content with their own network of connections. If the social graph is transparent and searchable, deeper analysis allows insights into relational behavior. Social media networks, by design, are no longer bounded to their own walled garden but allow users and organizations to use the API to create mobile apps and embed share and like buttons on third-party websites or simultaneously share content to other social networking sites.

In a relational approach, the unit of analysis is the tie between two actors, for example, the communication relationship between a government agency and individual citizens or the ties of citizens with other citizens. While government organizations might share one type of content-usually formal information or calls for action-citizens might alter the content, tag it so that others in their network can see it, or add contextual information to the original content. The analysis then focuses on the patterns of relationships that emerge while citizens are interacting with the content (Garton, Haythornthwaite, and Wellman 1997).

Combining relational analysis with attributes of actors, a more detailed understanding of who belongs to a subgroup and what the overall composition of the network looks like emerges (Freeman 1996; Kadushin 2012; Scott 2013). Measures that define the composition of the network include the following:

- The size as the total number of nominations an actor receives, measured as in-degree centrality (Borgatti 2005)

- The type of connections based on similarity among the actors' attributes or their other connections (see, e.g., McPherson, Smith-Lovin, and Cook 2001 on homogeneity of connections); citizens are more likely to make connections with their offline contacts and with those others in their network who share their political views or other similar attributes to avoid cognitive dissonance (Colleoni, Rozza, and Arvidsson 2014; Kossinets and Watts 2009)

- The multiplex nature of relationships (e.g., actors can talk about their professional tasks online, their experiences with a government service, and personal issues, such as child care) (Granovetter 1973; Mesch and Talmud 2006)

- The segmentation of the online constituency, measured using clustering coefficients to understand the number and types of cliques in a network that might make reach more difficult if information does not travel across cliques. Alternatively, it might be of interest to understand which (types of) actors are situated at the periphery of the network and might not have access to the same information as the majority of followers (Provan and Sebastian 1998). Reaching so-called peripheral groups can help public managers solve one of public administration's big questions. As Kirlin (1996) notes, issues of representativeness and reach of constituents are still among the "wicked" problems of public administration.

In contrast to the relational approach outlined earlier, a positional analysis defines which relative positions in a network are occupied as a result of their relationships (Barley 1990; White, Boorman, and Breiger 1976). Actors who forward content to their connections might also be willing to engage in higher levels of content reusefor example, write a blog post, embed links on a website, or pull social media content from a government agency into other direct marketing media, such as e-mail newsletters (Mergel 2013a).

Identifying roles in online networks helps decision makers understand who are the opinion makers in the network and who occupies other prominent positions, such as stars (an actor who is connected to many other isolated actors), bottlenecks (actors who decide which information to forward to an otherwise disconnected region of the network), or knowledge hubs (actors who in contrast to bottlenecks take on the role of receiving and distributing knowledge to the rest of the network). Other roles are dynamic and supportive by using their positions to forward information to a broader audience to amplify content. Centrality measures identify actors' roles in the network:

- Betweenness centrality measures the position of actors who lie in between network segments; information travels through them, reaching otherwise disconnected parts of the network (White and Borgatti 1994).

- Degree of centrality measures the number of connections of each actor. This measure can be separated into the number of outgoing (out-degree) and incoming (in-degree) ties so that knowledge hubs can be identified (Everett and Borgatti 1999; Tsai 2001).

To illustrate relational and positional factors, the network diagram in figure 1 shows two segments of a network that are connected

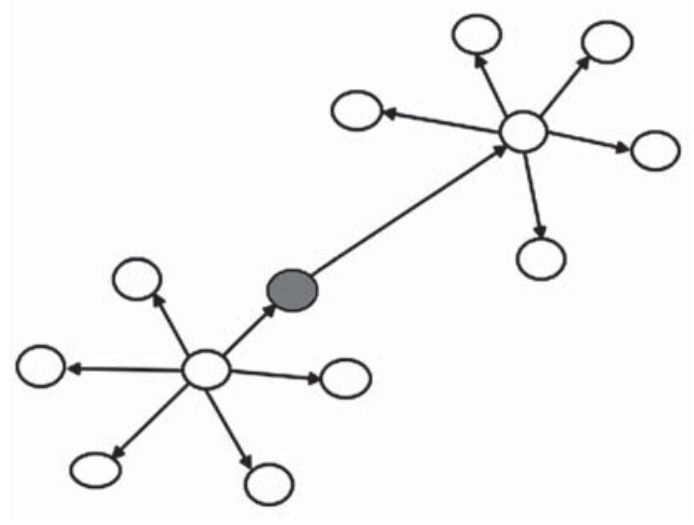

Figure 1 Relational and Positional Network Approach to Understand Social Media Networks 
through one central actor. The central actor receives content from the lower part of the network but only shares content with the upper part of the network (note the directions of the arrows).

pay attention to the data that they can collect and not the data that they should include. In addition, algorithms used to run analytics on extremely large social media data sets focus on regions in the data set with significant probability instead of peaks in the data or

Previous research has shown that ignoring or misinterpreting the composition, attributes, and attitudes of a follower network can be damaging for organizations and may lead to online firestorms. For example, the misguided belief that citizens love the New York City Police Department (NYPD) led the NYPD's public affairs officer to launch a Twitter campaign asking citizens to provide their positive experiences with the police department using the hashtag \#myNYPD. The organization's perceptions about its reputation differed widely from citizens' actual interactions with officers and resulted in a worldwide response of negative photographs showing police brutality (Jackson and Welles 2015). In this case, social media analytics of depth and breadth, as suggested by Kagarise and Zavattaro, would have shown that NYPD's social media efforts worked to increase the number of interactions but would not have helped social media managers understand their negative impact on the community.

\section{Limitations of Collecting and Analyzing Social Media Data}

Social media data are often described as providing unprecedented access to and insights into large-scale and highly detailed human behavior. Severo, Feredj, and Romele (2016) suggest that social media data can provide faster and more accurate insights that can help policy makers improve decision making. Scholars in other fields, including sociology, information sciences, and communication sciences, have established relational analysis of social media data. However, all disciplines are currently grappling with data collection issues, limitations of the data sets, and the application of network algorithms that were designed for much smaller data sets (Olmedilla, Martínez-Torres, and Toral 2016). Representativeness issues can limit and bias data collection efforts and challenge analytical reliability. Other limitations include problems with the use of existing network analytical algorithms designed for small data sets. Furthermore, combining social media data sets with administratively collected data poses privacy issues in the public sector. Therefore, the insights that can be gained by practitioners remain limited for practical reasons of data collection and analysis and for reasons of data biases (Mergel, Rethemeyer, and Isett 2016; Olteanu 2016).

Social media data are not representative of the population as a whole. A recent Pew Research Center study concludes that " $[\mathrm{i}] \mathrm{t}$ is important to remember that the people who take the time to post and talk about any subject—political or otherwise-on Twitter are a special group. Their conversations are not representative of the views or behaviors of the full Twitterverse. Moreover, Twitter users are only 18 percent of Internet users and 14 percent of the overall adult population" (Blank 2016; see also Smith et al. 2014). When using social media data to predict election results or the impact of a social policy on the whole population, practitioners will fall victim to the so-called streetlight effect (Bimber 2015): analysts tend to patterns that obviously stand out (Demirdjian et al. 2005). This means that the larger the data set, the more likely it is that patterns will be discovered.

Further, Lazer (2015) raises issues around construct instability in social media data that might not be easily mapped to administratively designed constructs of an interaction between a government organization and citizens. While we assume that constructs are stable over time, they might be dynamic and messy in social media data. This makes it difficult to combine or map administratively collected data (structured, stable) with unstructured dynamic social media data (Margolin et al. 2013). Some issues have to do with time delays and the accuracy of administratively collected statistics, publication delays, or missing data. Others have to do with the ability of citizens to opt out of automatic geotagging, which makes the so-called passive collection of social media data difficult for some nodes in the network. To overcome these issues, Lazer points to O'Brien, Sampson, and Winship (2015), who suggest studying small data paradigms using high-quality samples.

The practical challenge remains the lack of human and automated analytical skills in government. This is not to say that the current expertise is insufficient but rather that government budgets often lack professional development dollars for advanced computational skills or the resources to acquire state-of-the art computational social science solutions that have become part of the business analysis toolkits of social media managers in the private sector (Lazer et al. 2009). However, recent developments in the United States and the United Kingdom have shown that specialized teams of social and behavioral data scientists have been put in place to analyze big and social media data sets and to draw conclusions about the potential social impact of online behavior (see, e.g., https://sbst. gov/). However, there are lighthouse social media metrics projects that government practitioners can learn from, such as the Centers for Disease Control and Prevention's Digital Media Metrics Dashboard (https://www.cdc.gov/metrics/), or the General Services Administration's DigitalGov University on social media metrics (https://www.digitalgov.gov/category/socialmedia/).

\section{Conclusions}

Social media managers and public managers are facing the challenge of understanding whether their implementation of innovative forms of online communication has an impact on how citizens perceive government. While public sector organizations have trouble measuring performance such as return on investment, other forms of evidence are available to help them understand their impact on the communities they are serving.

This article offers an alternative to the common evidentiary approach of using data that are provided for free by social media platform providers. The measures suggested here complement 
those proposed by Kagarise and Zavattaro, who include in their social media impact analysis counts of how often a post is shared among citizens or how citizens are engaging with online government information by counting the number of comments or positive emoji left under a post. These measures are easily accessible even in a budget-conscious environment. Those responsible for social media in an organization tend to fall victim to the most obvious and loud voices in their social media time line. Those voices stick out and others might not be heard. This is partly attributable to the fact that analysts tend to pay attention to the data that they can collect and not the data that they should include.

As public managers prepare the promotion of new policies or programs, additional measures of social media impact are necessary. They need to focus on the inherent features of online social networks to understand whether all constituencies and stakeholders are being reached and what behavioral changes might result from social media interactions. These measures can help practitioners assess the potential impact of policy changes on their constituents or the mood in the network of subgroups. These holistic measures can prepare them for potential surprises as described in the \#myNYPD case, in which public affairs officers had no insights into how their organization is perceived by the public.

The suggestions presented in this article propose a paradigm shift toward a holistic view of social media data interactions and a change in how practitioners and scholars collect, analyze, and interpret evidence derived from social media. The insights provided here go beyond the current practices of public affairs and communication professionals as they are presented by Kagarise and Zavattaro. Applying a holistic approach has implications for other emergent concepts in the public sector, such as smart government approaches, the collection of behavioral and environmental data through sensors across cities, ad hoc use of crowdsourcing approaches, and other innovative forms of citizen interaction with government. Public administration as a field needs to engage diverse sets of evidence, apply computational social science approaches to collect and analyze the evidence, and allow for multiple perspectives to interpret the resulting data.

\section{References}

Barley, Stephen R. 1990. The Alignment of Technology and Structure through Roles and Networks. Administrative Science Quarterly 35(1): 61-103.

Bekkers, Victor, Arthur Edwards, and Dennis de Kool. 2013. Social Media Monitoring: Responsive Governance in the Shadow of Surveillance? Government Information Quarterly 30(4): 335-42.

Bimber, Bruce. 2015. What's Next: Three Challenges for the Future of Political Communication Research. In New Technologies and Civic Engagement: New Agendas in Communication, edited by Zuniga Navajas Homero Gil de, 215-23. New York: Routledge.

Blank, Gary. 2016. The Digital Divide among Twitter Users and Its Implications for Social Research. Social Science Computer Review. Published electronically on October 12. https://doi.org/10.1177/0894439316671698.

Borgatti, Stephen P. 2005. Centrality and Network Flow. Social Networks 27(1): 55-71.
Boyd, Danah M., and Nicole B. Ellison. 2007. Social Network Sites: Definition, History, and Scholarship. Journal of Computer-Mediated Communication 13(1): 210-30.

Campbell, David A., Kristina Lambright, and Christopher J. Wells. 2014. Looking for Friends, Fans, and Followers? Social Media Use in Public and Nonprofit Human Aervices. Public Administration Review 74(5): 655-63.

Colleoni, Elanor, Alessandro Rozza, and Adam Arvidsson. 2014. Echo Chamber or Public Sphere? Predicting Political Orientation and Measuring Political Homophily in Twitter Using Big Data. Journal of Communication
64(2): 317-32.

Daniell, Katherine A., Alec Morton, and David Ríos Insua. 2016. Policy Analysis and Policy Analytics. Annals of Operations Research 236(1): 1-13.

De Marchi, Giulia Lucertini Giada, and Alexis Tsoukiàs. 2016. From Evidence-Based Policy Making to Policy Analytics. Annals of Operations Research 236(1): 15-38.

Del Vicario, Alessandro Bessi Michela, Fabiana Zollo, Fabio Petroni, Antonio Scala, Caldarelli Guido, H. Eugene Stanley, and Walter Quattrociocchi. 2016. The Spreading of Misinformation Online. Proceedings of the National Academy of Sciences of the United States 113(3): 554-59.

Demirdjian, David, Leonid Taycher, Gregory Shakhnarovich, Kristen Grauman, and Trevor Darrell. 2005. Avoiding the "Streetlight Effect": Tracking by Exploring Likelihood Modes. Paper presented at the 10th IEEE International Conference on Computer Vision, Beijing, China, October 17-21.

Driscoll, Kevin, and Shawn Walker. 2014. Big Data, Big Questions-Working within a Black Box: Transparency in the Collection and Production of Big Twitter Data. International Journal of Communication 8: 1745-64.

Ellison, Nicole B., and Danah M. Boyd. 2013. Sociality through Social Network Sites. In The Oxford Handbook of Internet Studies, edited by W. H. Dutton, 151-72. Oxford, UK: Oxford University Press.

Everett, Martin G., and Stephen P. Borgatti. 1999. The Centrality of Groups and Classes. Journal of Mathematical Sociology 23(9): 181-201.

Freeman, Linton C. 1996. Some Antecedents of Social Network Analysis. Connections 19(1): 39-42.

Garton, Laura, Caroline Haythornthwaite, and Barry Wellman. 1997. Studying Online Social Networks. Journal of Computer-Mediated Communication 3(1). http://onlinelibrary.wiley.com/doi/10.1111/j.1083-6101.1997.tb00062.x/full [accessed April 17, 2017].

Granovetter, Mark. 1973. The Strength of Weak Ties. American Journal of Sociology 78(6): 1360-80.

Greenwood, Shannon, Andrew Perrin, and Maeve Duggan. 2016. Social Media Update 2016. Pew Research Center, November 11. http://www.pewinternet. org/2016/11/11/social-media-update-2016/ [accessed April 17, 2017].

Grimmelikhuijsen, Stephan G., and Albert J. Meijer. 2015. Does Twitter Increase Perceived Police Legitimacy? Public Administration Review 75(4): 598-607.

Isett, Kimberley R., Brian W. Head, and Gary VanLandingham. 2016. Caveat Emptor: What Do We Know about Public Administration Evidence and How Do We Know It? Public Administration Review 76(1): 20-23.

Jackson, Sarah J., and Brooke Foucault Welles. 2015. Hijacking \#myNYPD: Social Media Dissent and Networked Counterpublics. Journal of Communication 65(6): 932-52.

Kadushin, Charles. 2012. Understanding Social Networks: Theories, Concepts, and Findings. New York: Oxford University Press.

Kane, Gerald C., Maryam Alavi, Labianca Giuseppe (Joe), and Stephen P. Borgatti. 2014. What's Different about Social Media Networks? A Framework and Research Agenda. MIS Quarterly 38(1): 274-304.

Kim, Gang-Hoon, Silvana Trimi, and Ji-Hyong Chung. 2014. Big Data Applications in the Government Sector. Communications of the ACM 57(3): 78-85. 
Kirlin, John J. 1996. The Big Questions of Public Administration in a Democracy. Public Administration Review 56(5): 416-23.

Kossinets, Gueorgi, and Duncan J. Watts. 2009. Origins of Homophily in an Evolving Social Network. American Journal of Sociology 115(2): 405-50.

Lavertu, Stéphane. 2016. We All Need Help: "Big Data" and the Mismeasure of Public Administration. Public Administration Review 76(6): 864-72.

Lazer, David. 2015. Issues of Construct Validity and Reliability in Massive, Passive Data Collections. http://citiespapers.ssrc.org/issues-of-construct-validity-andreliability-in-massive-passive-data-collections/ [accessed April 17, 2017].

Lazer, David, Alex Pentland, Lada A. Adamic, Sinan Aral, Albert-László Barabási, Devon Brewer, Nicholas Christakis, et al. 2009. Life in the Network: The Coming Age of Computational Social Science. Science 323(5915): 721-23.

Margolin, Drew, Yu-Ru Lin, Devon Brewer, and David Lazer. 2013. Matching Data and Interpretation: Towards a Rosetta Stone Joining Behavioral and Survey Data. Paper presented at the Seventh International AAAI Conference on Weblogs and Social Media, Cambridge, MA, July 8-10.

Maultasch, Oliveira Gustavo Henrique, and Eric W. Welch. 2013. Social Media Use in Local Government: Linkage of Technology, Task, and Organizational Context. Government Information Quarterly 30(4): 397-405.

McPherson, Miller, Lynn Smith-Lovin, and James M. Cook. 2001. Birds of a Feather: Homophily in Social Networks. Annual Review of Sociology 27: 415-44.

Mergel, Ines. 2013a. A Framework for Interpreting Social Media Interactions in the Public Sector. Government Information Quarterly 30(4): 327-34.

Mergel, Ines. 2013b. Social Media Adoption and Resulting Tactics in the U.S. Federal Government. Government Information Quarterly 30(2): 123-30.

Mergel, Ines. 2014. A Manager's Guide to Assessing the Impact of Government Social Media Interactions. Washington, DC: IBM Center for the Business of Government.

Mergel, Ines, R. Karl Rethemeyer, and Kimberley R. Isett. 2016. Big Data in Public Affairs. Public Administration Review 76(6): 928-37.

Mesch, Gustavo, and Ilan Talmud. 2006. The Quality of Online and Offline Relationships: The Role of Multiplexity and Duration of Social Relationships. The Information Society 22(3): 137-48.

Mossberger, Karen, Yonghong Wu, and Jared Crawford. 2013. Connecting Citizens and Local Governments? Social Media and Interactivity in Major U.S. Cities. Government Information Quarterly 30(4): 351-58.

National Institute of Standards and Technology (NIST). 2010. Guide to Protecting the Confidentiality of Personally Identifiable Information (PII): Recommendations of the National Institute of Standards and Technology. http://nvlpubs.nist.gov/nistpubs/Legacy/SP/nistspecialpublication800-122.pdf [accessed April 17, 2017].

O'Brien, Daniel, Robert J. Sampson, and Christopher Winship. 2015. Econometrics in the Age of Big Data: Measuring and Assessing "Broken Windows”: Using Large-Scale Administrative Records. Sociological Methododology 45(1): 101-47.
Olmedilla, M., M. R. Martínez-Torres, and S. L. Toral. 2016. Harvesting Big Data in Social Science: A Methodological Approach for Collecting Online UserGenerated Content. Computer Standards \& Interfaces 46: 79-87.

Olteanu, Alexandra. 2016. Probing the Limits of Social Data: Biases, Methods, and Domain Knowledge. Thesis, École Polytechnique Fédérale de Lausanne. http:// www.aolteanu.com/papers/EPFL_TH6892.pdf [accessed April 17, 2017].

Porumbescu, Gregory A. 2016. Linking Public Sector Social Media and E-Government Website Use to Trust in Government. Government Information Quarterly 33(2): 291-304.

Provan, Keith G., and Juliann G. Sebastian. 1998. Networks within Networks: Service Link Overlap, Organizational Cliques, and Network Effectiveness. Academy of Management Journal 41(4): 453-63.

Scott, John. 2013. Social Network Analysis. Thousand Oaks, CA: Sage Publications. Severo, Marta, Amel Feredj, and Alberto Romele. 2016. Soft Data and Public Policy: Can Social Media Offer Alternatives to Official Statistics in Urban Policymaking? Policy \& Internet 8(3): 354-72.

Smith, Marc A., Lee Rainie, Ben Shneiderman, and Itai Himelboim. 2014. Mapping Twitter Topic Networks: From Polarized Crowds to Community Clusters. Pew Research Center, February 20. http://www.pewinternet.org/2014/02/20/ mapping-twitter-topic-networks-from-polarized-crowds-to-community-clusters/ [accessed April 17, 2017].

Stamati, Teta, Thanos Papadopoulos, and Dimosthenis Anagnostopoulos. 2015. Social Media for Openness and Accountability in the Public Sector: Cases in the Greek Context. Government Information Quarterly 31(1): 12-29.

Tsai, Wenpin. 2001. Knowledge Transfer in Intraorganizational Networks: Effects of Network Position and Absorptive Capacity on Business Unit Innovation and Performance. Academy of Management Journal 44(5): 996-1004.

Ugander, Johan, Brian Karrer, Lars Backstrom, and Cameron Marlow. 2011. The Anatomy of the Facebook Social Graph. http://arxiv.org/pdf/1111.4503.pdf [accessed April 17, 2017].

Warren, Anne Marie, Ainin Sulaiman, and Noor Ismawati Jaafar. 2014. Social Media Effects on Fostering Online Civic Engagement and Building Citizen Trust and Trust in Institutions. Government Information Quarterly 31(2): 291-301.

White, Harrison C., Scott A. Boorman, and Ronald L. Breiger. 1976. Social Structure from Multiple Networks. I. Blockmodels of Roles and Positions. American Journal of Sociology 81(4): 730-80.

White, Harrison J., and Stephen P. Borgatti. 1994. Betweenness Centrality Measures for Directed Graphs. Social Networks 16(4): 335-46.

Wukich, Clayton, and Ines Mergel. 2016. Reusing Social Media Information in Government. Government Information Quarterly 33(2): 305-12.

Zavattaro, Staci M., P. Edward French, and Somya D. Mohanty. 2015. A Sentiment Analysis of U.S. Local Government Tweets: The Connection between Tone and Citizen Involvement. Government Information Quarterly 32(3): 333-41. 